\title{
IR SPECTRAL MAPPING OF THE MARTIAN SOUTH POLAR RESIDUAL CAP USING CRISM.
}

\author{
Jacqueline Campbel1 ${ }^{1,2}$, Panagiotis Sidiropoulos ${ }^{2}$, and Jan-Peter Muller ${ }^{2}$ \\ ${ }^{1}$ School of Environment and Technology, University of Brighton, East Sussex, BN2 4AT, j.campbell4@uni.brighton.ac.uk and \\ ${ }^{2}$ Imaging Group, Mullard Space Science Laboratory, University College London, Holmbury St Mary, Surrey, RH56NT, \\ p.sidiropoulos@ucl.ac.uk, j.muller@ucl.ac.uk
}

Commission VII, WG VI/4

KEY WORDS: Mars, SPRC, hyperspectral analysis, mineral and PAH mapping.

\begin{abstract}
:
Polycyclic aromatic hydrocarbons (PAHs) are considered to be important in theories of abiogenesis (Allamandola, 2011). There is evidence that PAHs have been detected on two icy Saturnian satellites using the Visual and Infrared Mapping Spectrometer (VIMS) on the Cassini spacecraft (Cruikshank et al., 2007). The hypothesised presence of PAHs in Mars south polar cap has not been systematically examined even though the Mars south polar cap may allow the preservation of organic molecules that are typically destroyed at the Martian surface by UV radiation (Dartnell et al. 2012). This hypothesis is supported by recent analyses of South Polar Residual Cap (SPRC) structural evolution (Thomas et al., 2009) that suggest the possibility that seasonal and long term sublimation may excavate dust particles from within the polar ice. Periodic sublimation is believed to be responsible for the formation of so-called "Swiss Cheese Terrain", a unique surface feature found only in the Martian south polar residual cap consisting of flat floored, circular depressions (Byrne, 2009). We show the first examples of work towards the detection of PAHs in Swiss Cheese Terrain, using data from the Compact Reconnaissance Imaging Spectrometer for Mars (CRISM), on board NASA's Mars Reconnaissance Orbiter (MRO). CRISM is designed to search for mineralogical indications of past and present water, thus providing extensive coverage of the south polar cap. In this work, we discuss whether CRISM infrared spectra can be used to detect PAHs in Swiss Cheese Terrain and demonstrate a number of maps showing shifts in spectral profiles over the SPRC.
\end{abstract}

\section{INTRODUCTION}

Since the fly-by of Mars by NASA's Mariner 4 in 1965, Mars has been frequently examined using high-resolution orbital imagery, and by spectrometers since Mariner 6 in 1969 (NASA,

2015). The dynamic polar regions of Mars have been systematically studied in detail in more recent years by the European Space Agency's (ESA) Mars Express launched in 2003 and by NASA's Mars Reconnaissance Orbiter (MRO) launched in 2005. MRO includes an imaging spectrometer, CRISM (Murchie et al., 2007) of resolutions down to $\approx 20 \mathrm{~m}$ (see later); the CTX Context Camera, obtaining 6m/pixel spatial resolution (Malin et al., 2007), and the High Resolution Imaging Science Experiment (HiRISE), capable of up to $0.25 \mathrm{~m} /$ pixel resolution (McEwen et al., 2007). Mars' south polar cap consists of a permanent $400 \mathrm{~km}$ diameter layer of solid $\mathrm{CO} 2$ and water ice [Vita-Finzi, 2005].

"Swiss Cheese Terrain" (SCT) is a unique surface feature found only in the Martian South Polar Residual Cap (SPRC). Its characteristic appearance (consisting of flat floored, circular depressions) is considered to be caused by seasonal differences in the sublimation rates of water and $\mathrm{CO} 2$ ice (Tokar et al., 2003). The permanent $\mathrm{CO}_{2}$ layer at the south pole allows SCT to build up over decades, resulting in dynamic features with a springtime scarp retreat rate of up to $8 \mathrm{~m} / \mathrm{Mars}$ year (Byrne and Ingersoll, 2002).

Sublimation may occur from the base of the $\mathrm{CO}_{2}$ ice sheet, resulting in the excavation of dust trapped within the ice; local ruptures at the surface of the ice allow $\mathrm{CO}_{2}$ gas jets to erupt, leaving a surface layer of newly exposed dust, thought to be responsible for the dark rims visible in Swiss Cheese sublimation features (Kieffer et al., 2006). Figures 1 and 2 show typical SCT morphology.

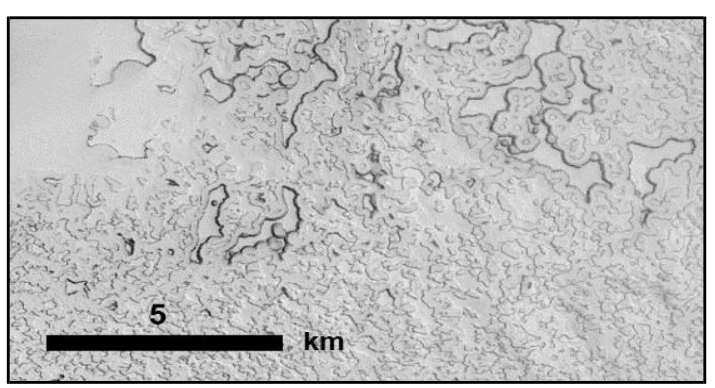

Figure 1: SCT Sublimation Features (CTX:B08_012572_0943_XI) 


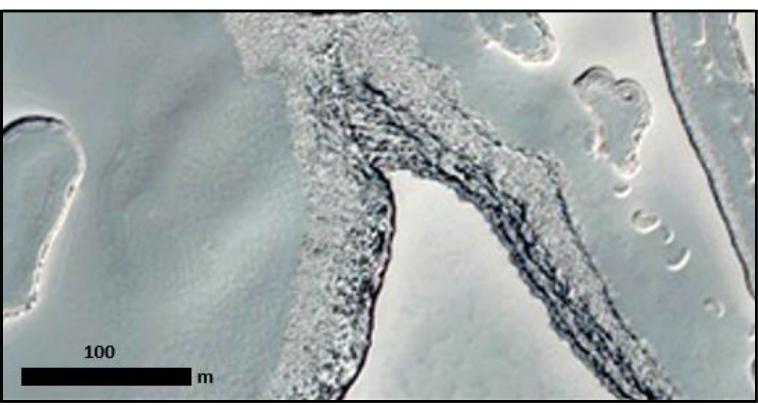

Figure 2: Close up of terraced scarp in SCT depression (HiRISE: PSP_004753_0930

The structural evolution of the SPRC has been investigated by Thomas et al. (2009), who identify various sub-units of SCT morphology with different rates of erosion, which may have provided protection for delicate organics.

In this work, we analyse Swiss Cheese Terrain features using data acquired by the Compact Reconnaissance Imaging Spectrometer for Mars (CRISM), which is on board NASA's Mars Reconnaissance Orbiter (MRO). CRISM is designed to search for mineralogical indications of past and present water, and has provided extensive coverage of the south pole. The targeted mode of the CRISM instrument uses the Optical Sensor Unit (OSU) to map key areas of mineralogical interest, $(<1 \%$ of the surface) where the OSU is scanned to remove almost all alongtrack motion, providing $10 \times 10 \mathrm{~km}$ footprints with $\sim 20 \mathrm{~m} /$ pixel spatial resolution, and spectral resolution of $6.55 \mathrm{~nm} /$ channel for the spectral range covering $362-3920 \mathrm{~nm}$ for 545 channels..

It should be noted that the CRISM instrument is not designed specifically for the identification of organics as a primary objective, however the large number of channels allows high sensitivity to carbonates which can be used to identify carbon rich material, which may allow for the identification of organic content (Murchie et al., 2007).

\section{POLYCYCLIC AROMATIC HYDROCARBONS}

The primary motivation for this investigation is the detection of polycyclic aromatic hydrocarbons (PAHs), a group of chemical compounds consisting of benzene rings of hydrogen and carbon that are particularly stable compared to aliphatic (non ring-like) hydrogen-carbon molecules (Carey, 2006). PAHs are considered to be important in theories of how life developed on Earth, and the search for organic molecules on Mars is important in ascertaining Mars' past conditions, and current habitability (Benner et al., 1998).

There are hundreds of species of PAH (Public Health England, 2008); they are abundant on Earth, and are a by-product of incomplete combustion of organic materials such as wood, tar and coal, and present in cooked food (Samanta et al., 2002).

PAHs occur not only on Earth, but throughout the universe, and have been found to coalesce in space within dust clouds; spectral signatures indicative of PAHs such as anthracene $\left(\mathrm{C}_{14} \mathrm{H}_{10}\right)$ and pyrene $\left(\mathrm{C}_{16} \mathrm{H}_{10}\right)$ were detected by Mulas et al. (2005) during their investigation of infrared emissions from the Red Rectangle Nebula., and there are close to 120 known interstellar species, which may account for up to $20 \%$ of total cosmic carbon (Allamandola, 2011). PAHs frozen within ice particles in dense molecular clouds in space undergo processing by ultraviolet light and cosmic rays to produce more complex species (Dartnell et al., 2012). These eventually rain down on primordial planets, either directly from planetary accretion discs, or are delivered on comets and meteorites (Allamandola, 2011).

The delivery of complex organic compounds to established, habitable planets via bolide impact is a very important concept in astrobiology, and could be instrumental in explaining abiogenesis. The presence of PAHs could indicate the degradation of organisms (Mckay et al., 1996) and could therefore be a biomarker for extinct or even extant life. The ability to identify PAHs using remote sensing could prove a critical tool in the search for extra-terrestrial organisms.

To date, the hypothesised connection of Martian Swiss Cheese Terrain and the presence of PAHs has not been systematically examined.

\section{METHODS}

\subsection{Selection of CRISM Scenes}

Only Full Targeted Resolution (FRT) CRISM products have been considered for study to try to maximise spatial resolution $(\sim 20 \mathrm{~m} / \mathrm{pixel})$ of small-scale rim features. Analysis of the SPRC has been carried out using HiRISE, CTX, MOC-NA and HRSC imagery to better constrain regions of interest, and select CRISM scenes for spectral analysis.

Due to inconsistent nomenclature and subcategories of sublimation features on the SPRC, it was not possible to simply search for CRISM images featuring SCT, and so a more systematic approach was required. The Arizona Lunar and Planetary Laboratory had labelled 50 HiRISE images as 'Swiss Cheese' or 'Swiss Cheese-Like' and by manually reviewing these images, it was clear that they covered a range of different SCT morphologies as described by Thomas et al., (2007). Starting with 50 HiRISE images identified as SCT, additional images from NASA's MOC-NA and CTX instruments were systematically analysed for widespread SCT coverage. Within this region, 72 FRT CRISM scenes were identified as containing SCT; these were arranged into groups of stacked images, resulting in 13 stacks each containing several FRT scenes taken over a period of 3 Martian years, totalling 55 images, which could then be examined for temporal and spatial spectral changes.

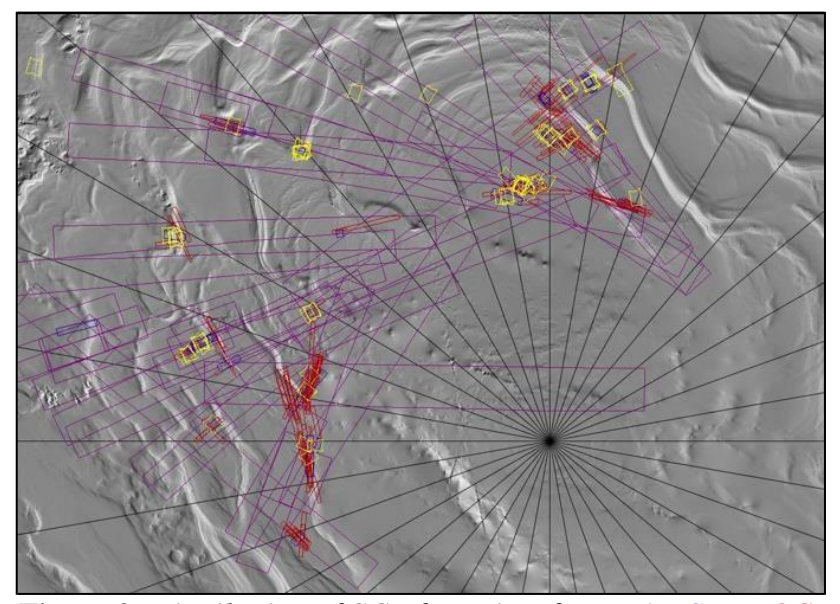

Figure 3: Distribution of SCT footprints from HiRISE, MOCNA, CTX and CRISM 
Figure 4 shows the areas of the SPRC covered with Swiss Cheese Terrain in rose, the location of the CTX footprint from Figure 1 in yellow, and the selected 55 CRISM scenes that will be used for spectral analysis in blue.

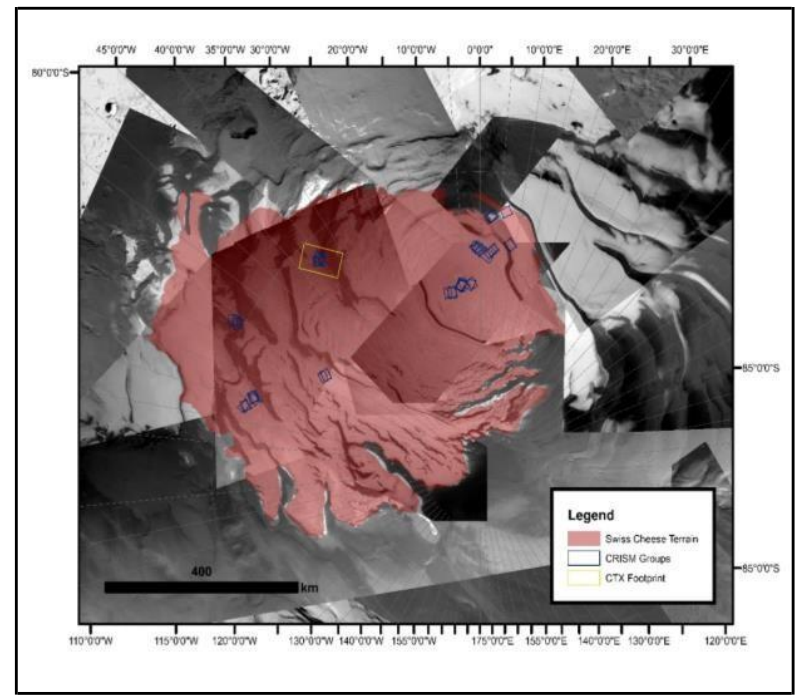

Figure 4: The Martian South Pole; the base map is comprised of HRSC (ND3 and ND4) images, with a resolution of $12.5 \mathrm{~m} /$ pixel. The images were manually co-registered using ArcMap. The area outlined in rose covers regions where SCT is visible in HRSC images. Overlaid are (1) an example of a CTX footprint, which is used to provide a higher resolution (6m/pixel) image of SCT (the yellow rectangle), and, (2) the 13 CRISM groups being used for SWIR spectral analysis (the blue rectangles).

\subsection{Data Processing and Calibration}

The CRISM Analysis Tool (CAT) plugin for ENVI software was used to process the 55 CRISM scenes with corrections for photometry, atmosphere, image artefacts, 'despiking' and 'destriping', and to generate summary products. 44 spectral summary products based on multispectral parameters are derived from reflectances for each CRISM observation that can be used as a targeting tool to identify areas of mineralogical interest for further analysis (Pelkey et al., 2007). Those of particular interest to this investigation are those which highlight carbon-rich materials, and $\mathrm{CO}_{2}$ and water ice in order to differentiate materials of astrobiological interest from the bulk of the SPRC.

\subsection{Spectral Mapping}

Figure 5 shows an RGB image of an area of SCT, while Figure 6 shows the same area in a false colour to highlight $\mathrm{CO}_{2}$ ice, water ice and carbonate overtones.

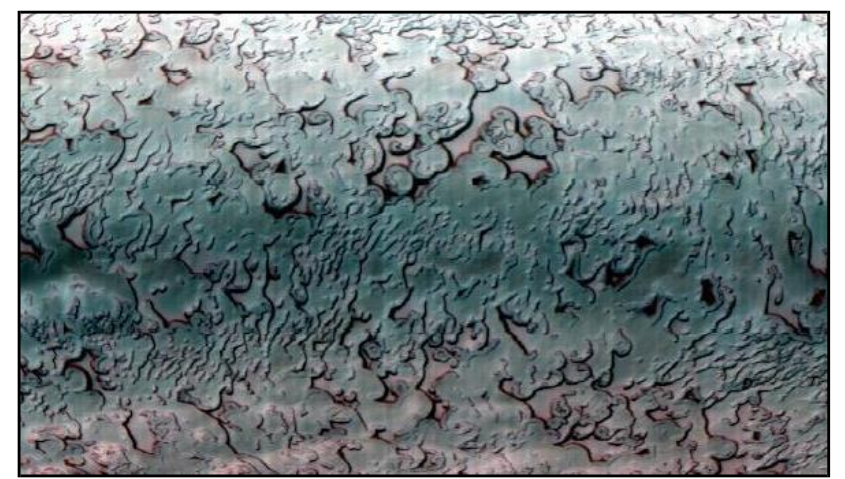

Figure 5: RGB visualisation of CRISM Scene 00005D24

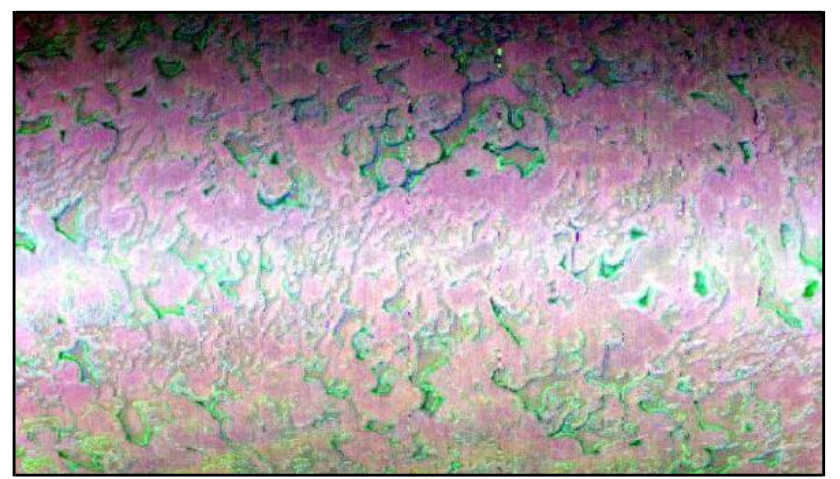

Figure 6: False colour visualisation of CRISM Scene 00005D2 using Pelkey's spectral summary products. Pelkey, et al., 2007). Red: $\mathrm{CO}_{2}$ ice, Green: Carbonate Overtones, Blue: Water ice

The areas highlighted in green around depression rims, indicating carbonate overtones, are those most likely to contain any possible signatures suggesting organics. Samples from the rims were taken using CAT's spectra collection function, using a minimum of 20 pixels/region of interest to minimise noise.

Figure 7 shows a region of interest, with dark scarps highlighted in blue, and an area of similar size from a featureless part of the SPRC in yellow. 


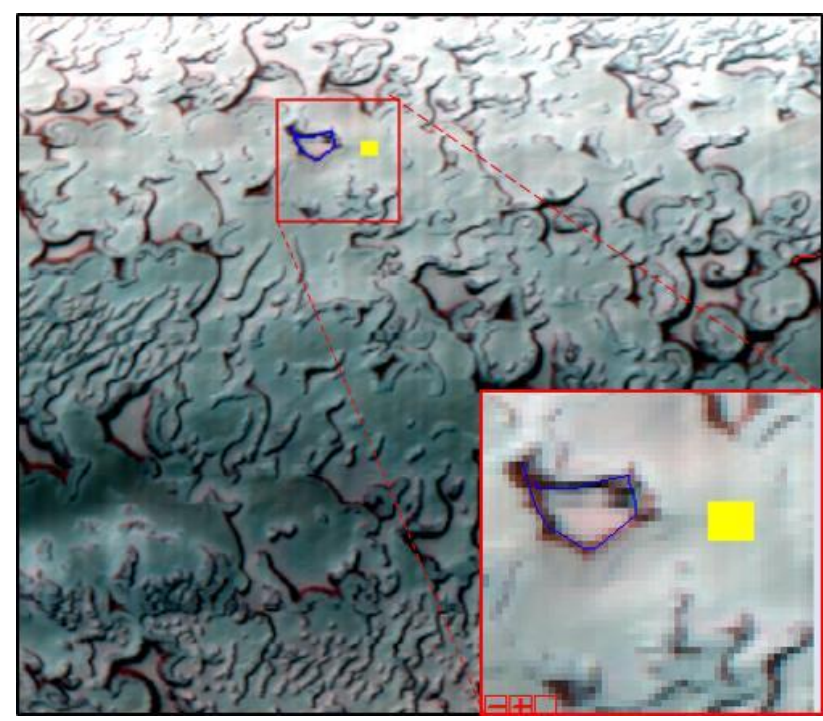

Figure 7: RGB visualisation of CRISM Scene 00005D24 with regions of interest inset

\section{RESULTS}

The scarp walls exhibit more spectral variation over a similar spatial area than mesas or depression floors. Figures 8 and 9 show the spectra for a featureless patch of ice and a dark rim.

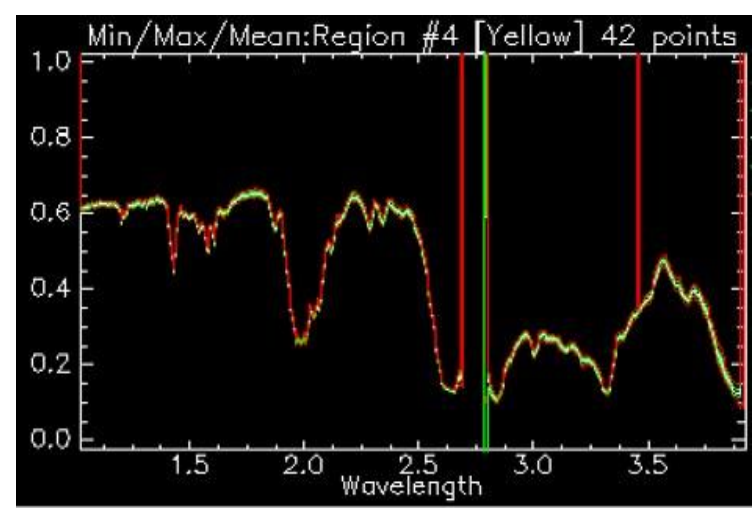

Figure 8: Maximum, minimum mean and standard deviations for the ROI in yellow in figure 7

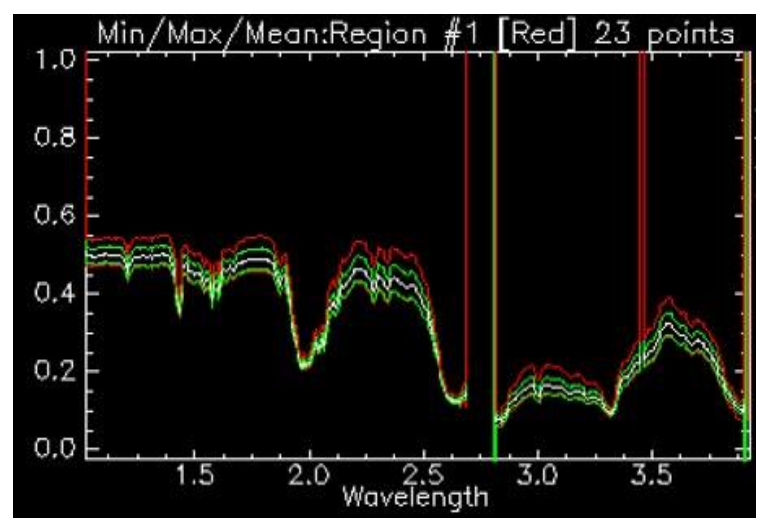

Figure 9: Maximum, minimum mean and standard deviations for the ROI in blue in figure 7

Work is now being undertaken to isolate peaks from the spectra from dark rims, and attribute known minerals to given features using the Peak-Fit software (SigmaPlot,2016). Remaining peaks are being compared to spectral libraries of PAHs to look for evidence of measureable organic content in SCT scarps. When spectral features associated with $\mathrm{CO}_{2}$ ice and known Martian regolith components, such as basalt, are removed from dust rim spectra, there remains a noticeable difference in the spectra of depression rims compared with depression floors and mesas. This is an ongoing project, and in the future we will have more detailed results to present.

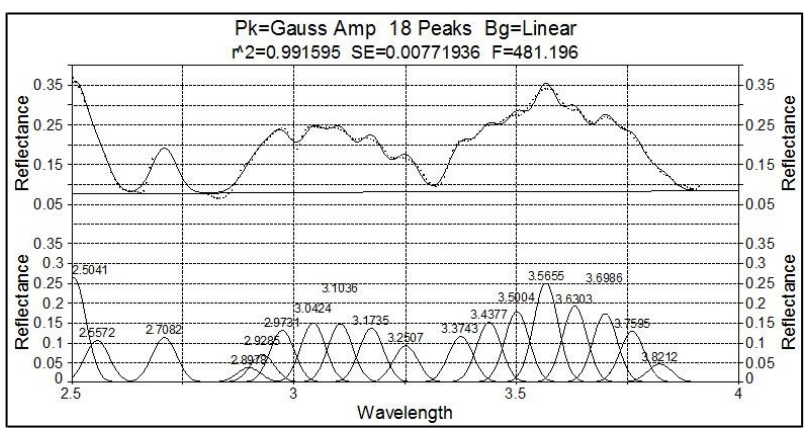

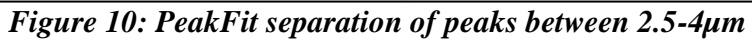

\section{ACKNOWLEDGEMENTS}

The research leading to these results has received partial funding from the STFC "MSSL Consolidated Grant" ST/K000977/1 and partial support from the European Union's Seventh Framework Programme (FP7/2007-2013) under iMars grant agreement $n^{\circ} 607379$.

\section{REFERENCES}

Allamandola, L.J. PAHs and Astrobiology. (2011) PAHS and the Universe, EAS publications series, 46. 305-317

Benner, S.A. Devine, K.G. Metveeva, L.N. Powell, D.H (1998). The missing organic molecules on Mars. PNAS. 97. 6. 24252430

Byrne, S. (2009). The Polar Deposits of Mars. Annual Review of Earth and Planetary Science. 37. 535-560

Byrne, S. Ingersoll, A.P. (2002) A sublimation model for the Martian polar swiss cheese features. Science. 299. 1051-1053

Carey, F.A. Organic Chemistry. $4^{\text {th }}$ Edition. McGraw-Hill. 398423

Cruikshank, D.P. et al. (2008), Hydrocarbons on Saturn's satellites Iapetus and Phoebe. Icarus, 193. 334-343

Dartnell, L. et al. (2012) Experimental determination of photostability and fluorescence-based detection of PAHs on the Martian surface. Meteoritics and Planetary Science. 47.5. 806819

Kieffer, H.H, Christensen. P.R, Titus. T.N. (2006). CO2 jets formed by sublimation beneath translucent slab ice in Mars' seasonal south polar ice cap. Nature. 443. 49-45.

Malin, M.C., Bell, J.F., Cantor, B.A., Caplinger, M.A., Calvin, W.M., Clancy, R.T., Edgett, K.S., Edwards, L., Haberle, R.M., James, P.B., Lee, S.W., Ravine, M.A., Thomas, P.C., Wolff, M.J., 2007. Context Camera investigation on board the Mars Reconnaissance Orbiter. J. Geophys. Res.: Planets 112. 5 
McEwen, A., Eliason, E., Bergstrom, J., Bridges, N., Hansen, C., Delamere, W., Grant, J., Gulick, V., Herkenhoff, K., Keszthelyi, L., Kirk, R., Mellon, M., Squyres, S., Thomas, N., Weitz, C., 2007. Mars Reconnaissance Orbiter's High Resolution Imaging Science Experiment (HiRISE). J. Geophys. Res.: Planets 112. 5

McKay, D. Gibson. E. (1996). Search for past life on Mars; possible relic biogenic activity in Martian meteorite ALH84001. Science. 273. 924-930.

Mulas, G. Malloci, C. Joblin, C. Toublanc, D. (2005). Estimated IR and phosphorescence emission fluxes for specific Polycyclic Aromatic Hydrocarbons in the Red Rectangle. Astronomy and Astrophysics. 446. pp.537-549

Murchie, S. Arvidson, R. Bedini, P. Beisser, K. Bibring, J-P. Bishop, J. Boldt, J. Cavender, P. Choo, T. Clancy, R.T. Darlington, E.H. Des Marais, D. Espiritu, R. Fort, D. Green, R. Guinness, E. Hayes, J. Hash, C. Heffernan, K. Hemmler, J. Heyler, G. Humm, D. Hutcheson, J. Izenberg, N. Lee, R. Lees, J. Lohr, D. Malaret, E. Martin, T. McGovern, J.A. McGuire, P. Morris, P. Mustard, J. Pelkey, S. Rhodes, E. Robinson, M. Roush, T. Schaefer, E. Seagrave, G. Seelos, F. Silverglate, P. Slavney, S. Smith, M. Shyong, W.J. Strohbehn, K. Taylor, H. Thompson, P. Tossman, B. Wirzburger, M. Wolff, M. (2007). Compact Reconnaissance Imaging Spectrometer for Mars (CRISM) on Mars Reconnaissance Orbiter (MRO). Journal of Geophysical Research. 112. E05S03, doi:10.1029/2006JE002682

NASA (2015) Mariner Missions. [ONLINE] $\mathrm{http}: / /$ science.nasa.gov/missions/marinermissions/ [Accessed 17 July, 2015]

Pelkey, S.M. Mustard, J.F. Murchie, S. Clancy, R.T. Wolff, M. Smith, M. Milliken, R. Bibring, J.P. Gendrin, A. Poulet, F. Langevin, Y. Gondet, B. (2007). CRISM multispectral summary products: Parameterizing mineral diversity on Mars from reflectance. Journal of Geophysical Research. 112. E08S14, doi:10.1029/2006JE002831

Public Health England. (2008). Polycyclic aromatic hydrocarbons (Benzo[a]pyrene). Toxicology Dept. Centre for Radiation, Chemicals and Environmental Hazards. Version 1.

Samanta, S.K. Singh, O.V. Jain, R.K. (2002). Polycyclic aromatic hydrocarbons: environmental pollution and bioremediation. Trends in Biotechnology. 20.6. 243-248

SigmaPlot. (2016). PeakFit Automated Peak Separation Analysis. http://sigmaplot.co.uk/products/peakfit/peakfit.php

Tokar R. L. Elphic, R.C. Feldman, W.C. Funsten, H. Moore, K.R. Prettyman, T. H. Wiens, R.C. (2003). Mars Odyssey neutron sensing of the south pole residual cap. Geophysical Research Letters. 30, 1677, 13

Thomas, P.C. et al. (2009) Residual south polar cap of Mars: Stratigraphy, history and impolications of recent changes. Icarus. 203. 2. 352-375.

Vita-Finzi C. (2005). Planetary Geology: An Introduction. Terra Publishing, 146-159. 